\title{
Sensing applications of long-period gratings in various fibre types
}

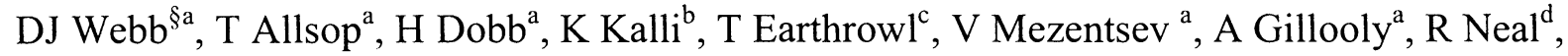 \\ I Bennion $^{\mathrm{a}}$ \\ ${ }^{a}$ Photonics Research Group, Aston University, Aston Triangle, Birmingham, B4 7ET, UK \\ ${ }^{b}$ Higher Technical Institute, Cyprus \\ c Clinical Biomedical Engineering Research Group, Aston University \\ ${ }^{\mathrm{d}}$ Dept. of Communications and Electrical Engineering, Faculty of Technology, University of Plymouth, UK
}

\begin{abstract}
In this paper we review the sensing features of long period Bragg gratings and report on studies aimed at optimising the sensitivities to various measurands by using different fibre geometries.
\end{abstract}

Keywords: optical sensor, long period grating, temperature, strain, bending, refractive index

\section{INTRODUCTION - COMPARISON OF LPGs AND FBGs}

In this paper we review the sensing capabilities of Long Period Gratings (LPGs) ${ }^{1}$ and Fibre Bragg Gratings (FBGs) in order to determine the areas where LPGs have advantages. We then go on to describe some recent research at Aston University seeking to capitalise on the particular features of LPGs.

\begin{tabular}{|c|c|c|c|c|}
\hline \multirow[t]{2}{*}{ Feature } & \multicolumn{2}{|c|}{ SMF Telecoms Optical fibre } & \multicolumn{2}{|c|}{ LPGs in other fibre types } \\
\hline & FBG & LPG & Maximum & Minimum \\
\hline Period & $0.5 \mu \mathrm{m}$ & $300 \mu \mathrm{m}$ & - & - \\
\hline Length & $5 \mathrm{~mm}$ & $5 \mathrm{~cm}$ & - & - \\
\hline Width of attenuation bands & $0.5 \mathrm{~nm}$ & $8 \mathrm{~nm}$ & $15 \mathrm{~nm}$ & $3 \mathrm{~nm}$ \\
\hline Number of attenuation bands & $1+$ harmonics & many & many & many \\
\hline Temperature sensitivity@1550 nm & $0.014 \mathrm{~nm} /{ }^{\circ} \mathrm{C}$ & $-0.41 \mathrm{~nm} /{ }^{\circ} \mathrm{C}$ & $+0.68 \mathrm{~nm} /{ }^{\circ} \mathrm{C}$ & $-0.56 \mathrm{~nm} /{ }^{\circ} \mathrm{C}$ \\
\hline Strain sensitivity@1550 nm & $1.2 \mathrm{pm} / \mu \varepsilon$ & $+0.9 \mathrm{pm} / \mu \varepsilon$ & $+4.0 \mathrm{pm} / \mu \varepsilon$ & $-6.1 \mathrm{pm} / \mu \varepsilon$ \\
\hline Bend sensitivity@1550nm & No & $+9.5 \mathrm{~nm} . \mathrm{m}$ & $+9.5 \mathrm{~nm} . \mathrm{m}$ & $-7.4 \mathrm{~nm} \cdot \mathrm{m}$ \\
\hline Index sensitivity@1550 nm & No & $-465 \mathrm{~nm}$ & $+5000 \mathrm{~nm}$ & $-2400 \mathrm{~nm}$ \\
\hline
\end{tabular}

Table 1. Comparison of typical properties of FBGs and LPGs

The sensing features of FBGs and LPGs are summarised in Table 1, though it must be born in mind that the sensitivities quoted for the LPG in SMF are typical values and can vary greatly with cladding mode order. Compared to FBGs, the main disadvantages of LPGs are: generally broader absorption bands, reducing the number of sensors that can be interrogated by a single source as well as limiting the precision with which the central wavelength can be monitored; complex spectra, usually consisting of several absorption bands, each with different sensitivities; and sensitivity to many measurands, which can increase cross-sensitivity problems. On the other hand, the advantages of LPGs include: ease of fabrication, permitting the use of simple point-by-point photoinscription or an electric arc; sensitivity to external refractive index and curvature; multiple absorption bands which may exhibit radically different measurand sensitivities opening up the possibility of multi-parameter sensing with a single device; and strong dependence of the sensing characteristics on the optical properties of the core and cladding, and of the cladding mode order ${ }^{1}$.

The last point is, we feel, particularly important. It offers the possibility of tailoring the sensing characteristics of the LPG to provide, for example, enhanced strain sensitivity while minimising temperature cross-sensitivity. The LPG's

${ }^{\S}$ Email: d.j.webb@aston.ac.uk 
behaviour can be controlled by adjusting the material properties (by using different dopants in the silica, or fibres constructed from other substances, e.g. polymers), by varying the waveguide geometry (e.g. using D-shaped, multi-clad or photonic crystal fibre) or by using different cladding mode orders.
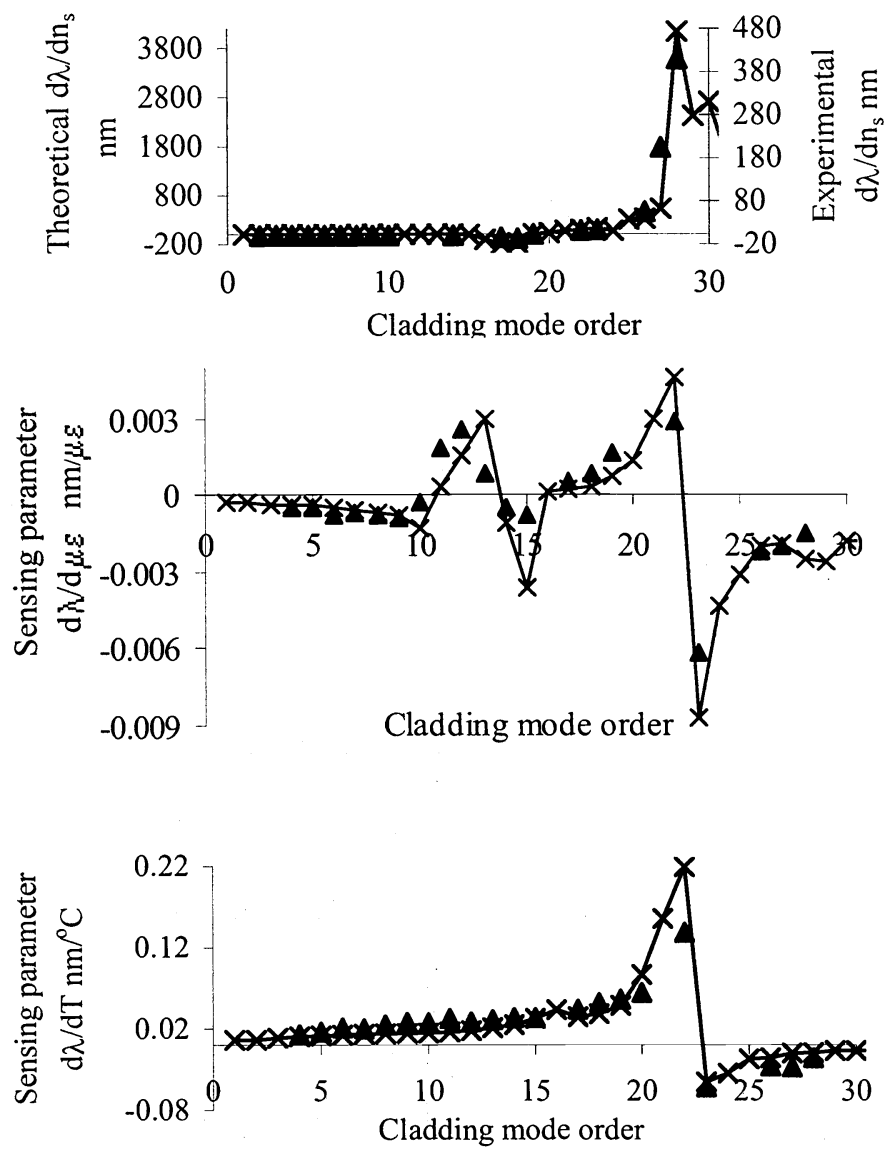

Figure 1. Experimental (triangles) and theoretical (crosses) sensitivity. TOP: index sensitivity of PTL fibre; MIDDLE: strain sensitivity of W-fibre; BOTTOM: temperature sensitivity of W-fibre.

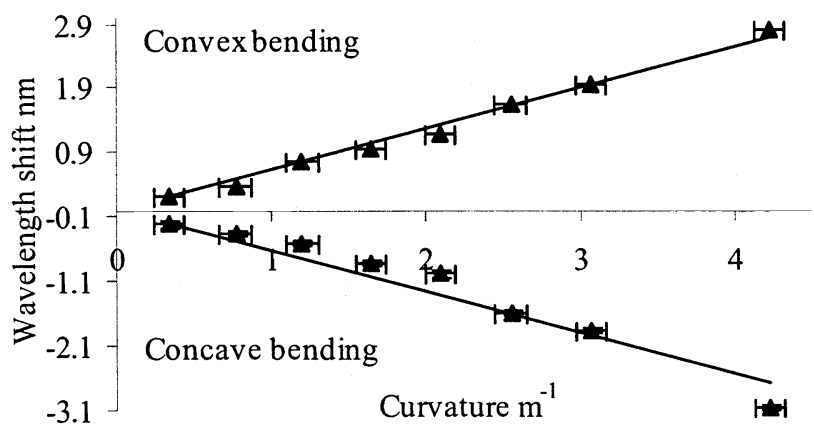

Figure 2. Wavelength shift due to concave and convex curvature of a D-shaped fibre.

\section{LPG CHARACTERISATION}

To date, we have not had the opportunity to design and produce fibres for specific sensing applications. Instead, we have explored a range of commercially available fibre types to determine their sensitivity to the various measurands: strain, temperature, external refractive index and bending.

2.1 Comparison of progressive three layered, wprofile and standard single mode fibre

An indication of the advantages to be gained by modifying the fibre structure is provided by our recent study into the sensing characteristics of LPGs recorded in progressive three layered (PTL) fibre, W-profile fibre and standard $\mathrm{SMF}^{2}$. Some example data is shown in Figure 1. The upper graph demonstrates that for the PTL fibre, the LPG is insensitive to external index for coupling to cladding modes with a radial order less than 15 . This is a useful property if the LPG is to be embedded in some other dielectric material and a shift in the attenuation and position is not wanted. The lower pair of graphs depict the strain and temperature dependencies of LPGs in the W-profile fibre. Comparing for example the bands associated with the $13^{\text {th }}$ and $14^{\text {th }}$ cladding modes, we see similar temperature sensitivities but strain sensitivities of opposite sign. Such a pair of features can be used to recover both temperature and strain information from a single LPG in an extremely well conditioned way.

\subsection{D-shaped fibre}

The spectral behaviour of LPGs in D-shaped fibre is rather complex. As a result of the fibre asymmetry, many of the coupling constants relating to the different cladding modes are small when the fibre is straight; consequently, many of the attenuation bands that might otherwise be expected are either absent or small ${ }^{3}$. When used to measure curvature, the bending of the fibre causes some of the initially large coupling constants to decrease, while others increase. As a result of this, as the fibre is bent some attenuation bands fade away, others appear while yet others are present for a large range of curvatures. The situation is further complicated by the fact that many of the bands that appear when the fibre is bent split into two, which separate with increased bending. This splitting is not birefringence related. 
The asymmetric structure complicates the theoretical modelling of LPGs in D-shaped fibre. We have resorted to using finite element analysis to calculate the wavelength dependence of the propagation constants of the various modes and have dealt with bending of the fibres using a conformal mapping technique ${ }^{3}$.

A potentially useful feature of LPGs in D-shaped fibre arises from the fibre asymmetry. In a circularly symmetric fibre, bending the fibre in any direction produces the same effect on the LPG's attenuation bands. In the case of the D-shaped fibre, when the fibre is bent so that the flat of the $\mathrm{D}$ is concave, the core, which lies near the flat is under compression, conversely, a bend making the flat of the $\mathrm{D}$ convex leads to axial tension in the core. Consequently, the shift in the attenuation band as a result of bending has a different sign depending on the direction of the bend. Note that the bend sensitivity is not the same for the two directions; in one case the strain in the core adds to the usual bend induced shift, while for the other direction it subtracts - see Figure 2 for an example.


Figure 3. Experimental characterisation of sensitivity of LPG in PCF to strain (upper) and curvature (lower). Arrows indicate the direction of increasing value from the nominal curve (bold).

\subsection{Photonic Crystal Fibre (PCF)}

Photonic crystal fibre is currently arousing great interest. From the perspective of LPGs, an attractive possibility is that by altering the geometry of the holes in the cladding one has some freedom to tailor the dispersive properties of the propagating modes without the need to change the glass material itself. This may greatly increase the ease of production of customised LPG sensors written in PCF.

Very few results of studies of LPGs in PCF have yet been reported, though already some intriguing behaviour has been seen. Most PCF is not photosensitive and hence the electric arc approach has been used to record LPGs. The first report of such devices noted that the LPG bands studied had an extremely low temperature sensitivity ${ }^{4}$. Such behaviour is very useful when other parameters are being sensed; indeed cross-sensitivity to temperature is a perennial problem for many sensing technologies. We have also used the electric arc technique for LPG fabrication and have carried out measurements of the sensitivity to strain, bending and temperature. Our results for strain and bending are shown in Figure 3 and yield sensitivities of 2.0 $\mathrm{pm} / \mu \varepsilon$ and $3.7 \mathrm{~nm} . \mathrm{m}$, respectively. It is particularly interesting to note that although the parameters of our fibre are different from those in reference 6 , we also were able to observe no temperature shift over a $70{ }^{\circ} \mathrm{C}$ range, which places an upper limit on the sensitivity of $10 \mathrm{pm} /{ }^{\circ} \mathrm{C}$.

\section{EXAMPLE APPLICATION - RESPIRATORY MONITORING}

As an example of how some of the issues discussed above inform the design of a sensor, we will briefly describe our current work on respiratory monitoring. The impetus behind this work is a clinical need for a portable system that can provide information on the contributions to the process of respiration from various regions of the lungs. Currently, respiration is almost always assessed by a measurement of total flow rate or exhaled volume made at mouth. The very few exceptions to this either provide information on the motion of a small number points on the abdomen, or else are imaging systems that require the patient to remain within a fixed measurement volume.

The approach we are developing is based on using an array of curvature sensors to reconstruct the shape of the abdomen and the changes in shape induced by respiration. Ultimately the sensors will be incorporated in a wearable skin-tight vest, but at the current state of research we are using discrete, packaged sensors to enable us to investigate the optimum placement. Figure 4 shows a photograph of an individual sensor along with an array spread across a resuscitation training mannequin, which has an inflatable "chest" capable of generating repeatable curvature changes typical of those displayed by a person. 

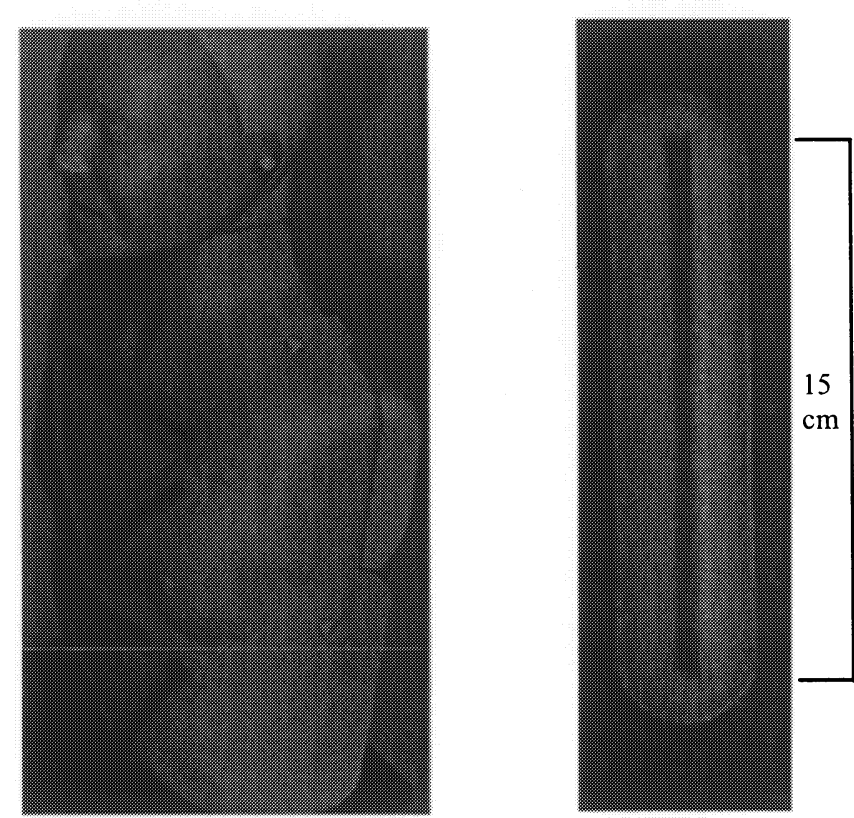

Figure 4. Respiratory monitoring using LPGs. RIGHT: packaged sensor; LEFT: sensor testing using mannequin.
The LPGs are currently embedded in $2 \mathrm{~mm}$ thick silicon rubber along with a short length of carbon fibre tow, see Figure 4 RIGHT. The first problem that faced us in the design was the sensitivity of LPGs to external index, which can result in a significant wavelength shift when the device is embedded, and can also contribute to the temperature sensitivity. The problem was solved using PTL fibre, where the first 15 bands are insensitive to the external index (see Figure 1). The next issue was temperature sensitivity. This varies between the different cladding modes and we have chosen one where the effect is relatively small; for the curvature accuracy needed, the device has a useable temperature range of about $3{ }^{\circ} \mathrm{C}$. This is satisfactory for initial studies where the device will be in contact with the skin, but we would prefer the sensitivity to be less. Part of the motivation for our work on LPGs in PCF comes from this kind of requirement.

We are still left with the issue of strain sensitivity. Currently we are not able to remove this problem through LPG design, however for this application the issue is not so much strain sensitivity as the sensitivity of the LPG to the stress placed on the sensor by the breathing process. By incorporating the carbon fibre tow

we have introduced an axially rigid structure that nevertheless is still very flexible. The tow ensures that the kinds of stresses likely to be experienced produce negligible strain levels in the fibre.

By use of the mannequin we have demonstrated that the sensors can provide sufficient curvature resolution; preliminary work on shape reconstruction (see Figure 4. LEFT) has suggested that an array of 20-25 sensors should be sufficient to recover the abdomen shape with sufficient precision; and we have developed a multiplexing system capable of handling this many LPGs ${ }^{6}$. The next stage of the work will involve making a prototype vest capable of accepting 25 sensors to enable us to collect data from human subjects to prove the clinical viability of the approach.

\section{CONCLUSION}

In this paper we have tried to highlight the features of LPGs that we feel make them attractive from a sensing point of view and we have described some of our recent research aimed at evaluating and exploiting these advantages.

\section{REFERENCES}

1 S W James and R P Tatam, "Optical fibre long-period grating sensors: characteristics and application," Meas. Sci. Technol., 14 (5) R49-61, 2003.

2 T Allsop, D J Webb and I Bennion, "A comparison of the sensing characteristics of long period gratings written in three different types of fiber," Opt. Fiber Tech. (9), pp. 210-223, 2003.

3 T Allsop, A Gillooly, V K Mezentsev, T Earthgrowl-Gould, R Neal, D J Webb and I Bennion, "The Spectral Characteristics of Long Period Gratings Written in D-Shaped Optical Fiber as Bending Sensors," Proceedings of the 16th International Conference on Optical Fibre Sensors (OFS '2003), IEICE, Japan , pp. 88-91, 2003.

4 G. Humbert, A. Malki, S. Fevrier, P. Roy and D. Pagnoux. "Electric arc-induced long-period gratings in Ge-free airsilica microstructure fibres," Electron. Lett. 2003, 39 (4), pp 349-350.

$5 \mathrm{~T}$ Allsop, T Earthrowl-Gould, D J Webb and I Bennion, "Embedded progressive-three-layered fiber long-period gratings for respiratory monitoring," J. Biomed. Opt. 8 (3) , pp. 552-558, 2003.

6 T Allsop, T Earthrowl-Gould, R Reeves, D J Webb and I Bennion, "The interrogation/multiplexing of long period gratings curvature sensors using derivative spectroscopy technique based upon Fiber Bragg Gratings", Meas. Sci. Tech. 15 (1), pp. 44-48, 2004. 